\title{
Neurotization Improves Contractile Forces of Tissue-Engineered Skeletal Muscle
}

\author{
VIKAS DHAWAN, M.D., Ph.D., ${ }^{1}$ IAN F. LYTLE, M.D., ${ }^{1}$ DOUGLAS E. DOW, Ph.D., \\ YEN-CHIH HUANG, Ph.D., ${ }^{2}$ and DAVID L. BROWN, M.D., FACS ${ }^{1}$
}

\begin{abstract}
Engineered functional skeletal muscle would be beneficial in reconstructive surgery. Our previous work successfully generated 3-dimensional vascularized skeletal muscle in vivo. Because neural signals direct muscle maturation, we hypothesized that neurotization of these constructs would increase their contractile force. Additionally, should neuromuscular junctions (NMJs) develop, indirect stimulation (via the nerve) would be possible, allowing for directed control. Rat myoblasts were cultured, suspended in fibrin gel, and implanted within silicone chambers around the femoral vessels and transected femoral nerve of syngeneic rats for 4 weeks. Neurotized constructs generated contractile forces 5 times as high as the non-neurotized controls. Indirect stimulation via the nerve elicited contractions of neurotized constructs. Curare administration ceased contraction in these constructs, providing physiologic evidence of NMJ formation. Histology demonstrated intact muscle fibers, and immunostaining positively identified NMJs. These results indicate that neurotization of engineered skeletal muscle significantly increases force generation and causes NMJs to develop, allowing indirect muscle stimulation.
\end{abstract}

\section{INTRODUCTION}

$\mathbf{T}$ ISSUE ENGINEERING IS A PROMISING APPROACH to the everincreasing need for treatment of tissue loss and endstage organ failure. In 1993, more than $\$ 400$ billion was spent, and more than 8 million procedures were performed to treat patients suffering from organ failure or tissue loss. ${ }^{1}$ A reliable and renewable source of skeletal muscle would be of great benefit to the patient who has lost muscle tissue or muscular function.

Previously, we have used a model of spontaneous angiogenesis that proved to be an excellent platform on which to generate in vivo 3-dimensional (3-D), vascularized, functional cardiac and skeletal muscle constructs. ${ }^{2,3}$ For these experiments, we adapted a method that creates a potential space around a vascular pedicle. ${ }^{4}$ The vascular pedicle consists of an artery, a vein, and the adherent connective tissue.
Over time, the void within the chamber spontaneously fills with neoangiogenic tissue, providing a well-vascularized platform to support the survival and growth of 3-D tissues.

Vascularization of our previous engineered skeletal muscle constructs permitted development of larger, stronger constructs than was possible in vitro. ${ }^{5}$ Despite this, the specific forces generated by native skeletal muscle were 4 orders of magnitude greater than the engineered constructs $\left(35.4 \mathrm{~N} / \mathrm{m}^{2}\right.$ vs. $239.7 \mathrm{kN} / \mathrm{m}^{2}$ for mouse extensor digitorum longus muscle). ${ }^{3}$ Because the expression of adult phenotype in muscle is directly related to the innervation and activation pattern of the muscle, ${ }^{6,7}$ we hypothesized that neurotization of the tissue-engineered skeletal-muscle constructs would result in the formation of neuromuscular junctions (NMJs), thereby improving force production and providing the means for indirect myocyte stimulation. Also, by introducing neural components to these axially vascularized

\footnotetext{
${ }^{1}$ Section of Plastic and Reconstructive Surgery, University of Michigan, Ann Arbor, Michigan.

${ }^{2}$ Department of Biomedical Engineering, University of Michigan, Ann Arbor, Michigan.
} 
constructs formed in vivo, we may provide them with their own neurovascular pedicle, thus facilitating their microsurgical transfer for future functional reconstruction purposes.

To produce a skeletal muscle construct with better functional potential, we sought to neurotize the developing skeletal muscle constructs in vivo. Previous studies of in vitro neurotized, cultured myocytes have described histological and chemical-biological interactions at the NMJ that lead to faster phenotypic maturity. ${ }^{8-10}$ Additionally, nerve-induced contractions of in vitro skeletal myotubes have been described. $^{11}$

\section{MATERIALS AND METHODS}

\section{Animal protocol}

The University Committee for the Use and Care of Animals granted institutional approval for animal use in accordance with the "Guide for the Care and Use of Laboratory Animals" ". Rats were housed individually in a restrictedaccess, pathogen-free facility. They had access to food and water ad libitum and were exposed to a 12-h light-dark cycle. All materials were purchased from Sigma (St. Louis, MO) unless otherwise specified.

\section{Isolation of skeletal myocytes}

Skeletal satellite cells were isolated from adult Fisher344 rat soleus muscle, a large skeletal muscle found in the lower leg, using an established method. ${ }^{13}$ The excised soleus muscles were placed in phosphate buffered saline (PBS) and then rinsed 3 to 4 times in sterile PBS to remove debris. The muscle was then cut into fine pieces and dissociated in a dissociation solution made of collagenase type II $(0.1 \%)$ and dispase $(0.05 \%)$ diluted in serum-free Dulbecco's modified Eagle medium (DMEM), for $3 \mathrm{~h}$ in a $37^{\circ} \mathrm{C}$ shaking water bath. After digestion, the solution was filtered through a $100-\mu \mathrm{m}$ filter and centrifuged at 2,500 revolutions per min for $6 \mathrm{~min}$ to pellet the cells. The supernatant was removed, and the cell pellet was resuspended in fresh growth medium (10\% heat-inactivated fetal bovine serum and $5 \mathrm{ng} / \mathrm{mL}$ fibroblast growth factor-2 in F12K nutrient mixture containing $100 \mathrm{U} \cdot 100 \mathrm{mg}^{-1} \cdot \mathrm{mL}$ penicillin streptomycin ${ }^{-1}$ and $2.5 \mu \mathrm{g} / \mathrm{mL}$ Fungizone). The cells were plated overnight, and the nonadherent cells were transferred to new plates to form additional cultures and increase our yield of skeletal cells. After the cells grew to $70 \%$ confluence, they were trypsinized with $1 \mathrm{~mL}$ of $0.025 \%$ trypsin per plate.

\section{Preparation of the chambers for implantation}

Silicone tubing $(3.2 \mathrm{~mm}$ inner diameter, $4.8 \mathrm{~mm}$ outer diameter, wall thickness $0.8 \mathrm{~mm}$; Cole Parmer Instrument Company, Vernon Hills, IL) was cut to lengths of $10 \mathrm{~mm}$, making an internal volume of $80 \mu \mathrm{L}$. A longitudinal slit was made down the length of each tube to allow it to be opened length-wise for placement around the intact femoral vessels. The previously isolated skeletal myocytes were suspended in a fibrinogen solution $(50 \mu \mathrm{L}$ of cells/culture medium and $20 \mu \mathrm{L}$ of $20-\mathrm{mg} / \mathrm{mL}$ fibrinogen Sigma) to obtain 6 million cells per chamber. The cell and fibrinogen suspension was pipetted into vertically oriented silicone tubes containing $10 \mu \mathrm{L}$ of $200-\mathrm{U} / \mathrm{mL}$ thrombin. Four chambers were prepared for each study group.

\section{Implantation of chambers}

Non-neurotized control procedure. Cell-seeded chambers were implanted into 4 adult F344 rats weighing approximately 250 to $300 \mathrm{~g}$ each. The animals were anesthetized using an intraperitoneal injection of $0.5 \mathrm{~mL}$ of sodium pentobarbital (Abbott Laboratories, Chicago, IL). Their groins were shaved, sterilely prepared, and draped. The right femoral vessels were exposed through a longitudinal incision made at the groin. With the aid of an operative microscope, the femoral artery and vein were separated from their surrounding tissues and the accompanying femoral nerve. Several small vascular branches to the thigh musculature were cauterized to isolate the femoral vessels over their lengths from the inguinal ligament to the branch point of the epigastric vessels. The previously prepared skeletal myocyte- and fibrin gel-filled tubes were placed around the femoral vessels by passing the intact vascular pedicle through the slit in the side of the silicone tubes. The wounds were closed in 2 layers.

Neurotized experimental procedure. On the left side of each of the above animals, the femoral vessels were exposed as above, and the nerve was dissected away from the vascular structures (Fig. 1A). The femoral nerve was then transected at an oblique angle so as to maximally expose the cross-sectional area of the proximal transected stump to the myoblasts in the chamber. The epineurium of the femoral nerve's proximal stump was then sutured to the connective tissue surrounding the femoral artery to position the severed nerve end into the middle of the developing construct (Fig. 1B, C). A myocyte- and fibrin gel-filled chamber was again placed around the intact femoral vessel and the proximal stump of the femoral nerve (Fig. 1B, D). The wound was closed in 2 layers, and the animal was allowed to recover. Constructs were implanted in this manner as the experimental procedure.

\section{Retrieval of chambers}

The constructs were allowed to mature in vivo for 4 weeks in this study, compared with the 3-week in vivo period of our earlier studies. ${ }^{3}$ The rationale for this was to increase the time needed for NMJ formation. After 4 weeks, the rats were re-anesthetized, and the chambers were isolated. The silicone tubes were opened along their longitudinal slit, and the patency of the vascular pedicles was confirmed using 


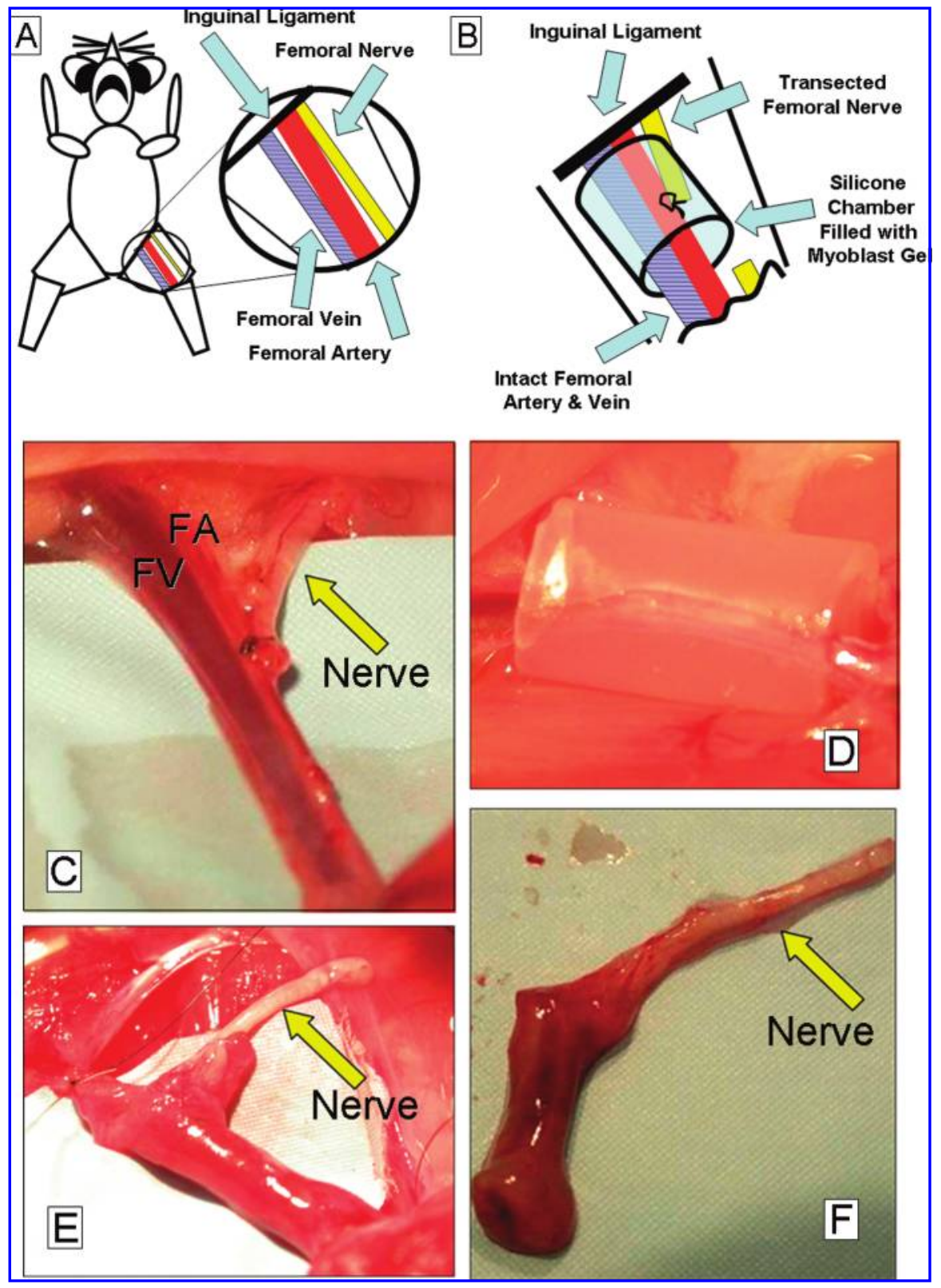

FIG. 1. In vivo vascularized, neurotized skeletal muscle construct development. (A) Drawing of rat in supine position with left groin dissected, demonstrating the inguinal ligament, femoral artery, femoral vein, and femoral nerve. (B) Drawing of a rat's left groin with femoral nerve transected and sutured to the adventitia of the femoral artery. The silicone chamber filled with myoblast and fibrin gel has been placed around the vascular pedicle and proximal stump of the femoral nerve. (C) Dissected rat groin with the femoral artery (FA) and femoral vein (FV). The proximal stump of the femoral nerve (arrow) has been sutured to the adventitia of the femoral artery. (D) Photo taken at the time of implantation. The silicone chamber, filled with myoblast and fibrin gel, has been placed around the intact femoral vessels and the proximal stump of femoral nerve (proximal vessels at left of picture, distal vessels at right). (E) Photo taken at the time of explantation. The silicone chamber has been removed, the proximal stump of femoral nerve (arrow) is well incorporated into the skeletal muscle construct, and proximal vessels (left) and distal vessels (right) are ligated. Note the newly formed cylinder of vascularized muscle tissue. (F) Photo of the neurotized skeletal muscle construct after harvest. Note the nerve entering the muscle construct (arrow). Color images available online at www.liebertpub.com/ten.

visual observation of blood flow through the vessels. The femoral artery and femoral vein were ligated and divided proximally and distally to the newly formed skeletal muscle constructs for the non-neurotized control side and the neurotized experimental side (Fig. 1E). The proximal stump of the femoral nerve was identified on the experimental side and was dissected free and divided proximally, leaving a proximal length attached to the construct (Fig. 1F). Once the skeletal muscle construct was freed, the severed nerve end was submerged in a solution of $50 \%$ polyethylene 
glycol (molecular weight 3000) in DMEM for $2 \mathrm{~min}$ to repair nerve membrane damage and prevent loss of nerve impulse conduction. ${ }^{14}$ The constructs were then placed into a 35-mm tissue culture plate coated with polydimethylsiloxane substrate (Dow Chemical Corporation, Midland, MI). DMEM with an antibiotic-antimycotic solution (Invitrogen Corporation, Auckland, New Zealand) was added to each plate. The contractility characteristics of the tissues were then determined, as described below.

\section{Functional assessment}

Field stimulation. The functional capabilities of the skeletal muscle constructs were measured according to active contractile force production ex vivo. Force testing was not performed in situ because the skeletal muscle construct was not fixed to any stable structure. After explanting the construct, one end was fixed to a stationary position and the other end of the construct was fixed to a force transducer. The method for evaluating the active contractile force of engineered muscle has been described in detail in previous work. ${ }^{13}$ Briefly, following explantation, each construct was placed in a 35-mm culture dish with DMEM culture medium, maintained at $37^{\circ} \mathrm{C}$ (Fig. 2). One end of the construct was fixed by a pin attached to the bottom of the dish and the other end of the construct was connected to a custom-built force transducer. A custom LabView software program (National Instruments, Austin, TX) running on a personal computer controlled the data input-output board. The LabView program and data input-output board generated output voltage signals that were amplified and delivered to the construct as direct field stimulation via 2 platinum electrode wires laying parallel to the constructs, one electrode on either side of the construct. Electrical stimulation was applied to the construct to generate contractile forces. Pulses having a 15 volt amplitude and a $1.2 \mathrm{~ms}$ width were used to determine peak twitch force. These parameters were chosen based on previous studies in which stimulation voltage was varied between 5 and 30 volts, to arrive at optimal stimulation parameters. In these past experiments, it was found that 15 volts was great enough to excite the tissue-engineered skeletal muscles to maximally contract, but not causing overt damage. Additionally, by using a narrower stimulation protocol, we decreased the amount of time that the constructs were stimulated in an ex vivo environment. Peak tetanic forces (forces generated while stimulating a constant, sustained contraction) were determined with a 1 second train of pulses (15 volt at $1.2 \mathrm{~ms}$ width) for which the pulse frequency was varied between 5 and $150 \mathrm{~Hz}$, to determine the maximal tetanic force generation per construct.

\section{Indirect stimulation}

Following direct field stimulation, each neurotized skeletal muscle construct was also stimulated indirectly, via the nerve. Needle electrodes were positioned so that the electrodes were touching the nerve, and an electrical stimulus of 1 to 5 volts was given for $1.2 \mathrm{~ms}$ (Fig. 3A, C). Twitch contractile forces were measured rather than tetanic forces secondary to concern of "burning out" the nerve with tetanic stimulation. To ensure that any resultant force from the intended indirect stimulation was not the result of direct field stimulation, the electrodes were then disconnected from the nerve and placed at a point equidistant from the muscle construct but not touching the nerve (Fig. 3B, D), and stimulation and force recording were again performed. Lastly, to ensure that any contractile forces caused by the indirect stimulation were due to intact neural communication with

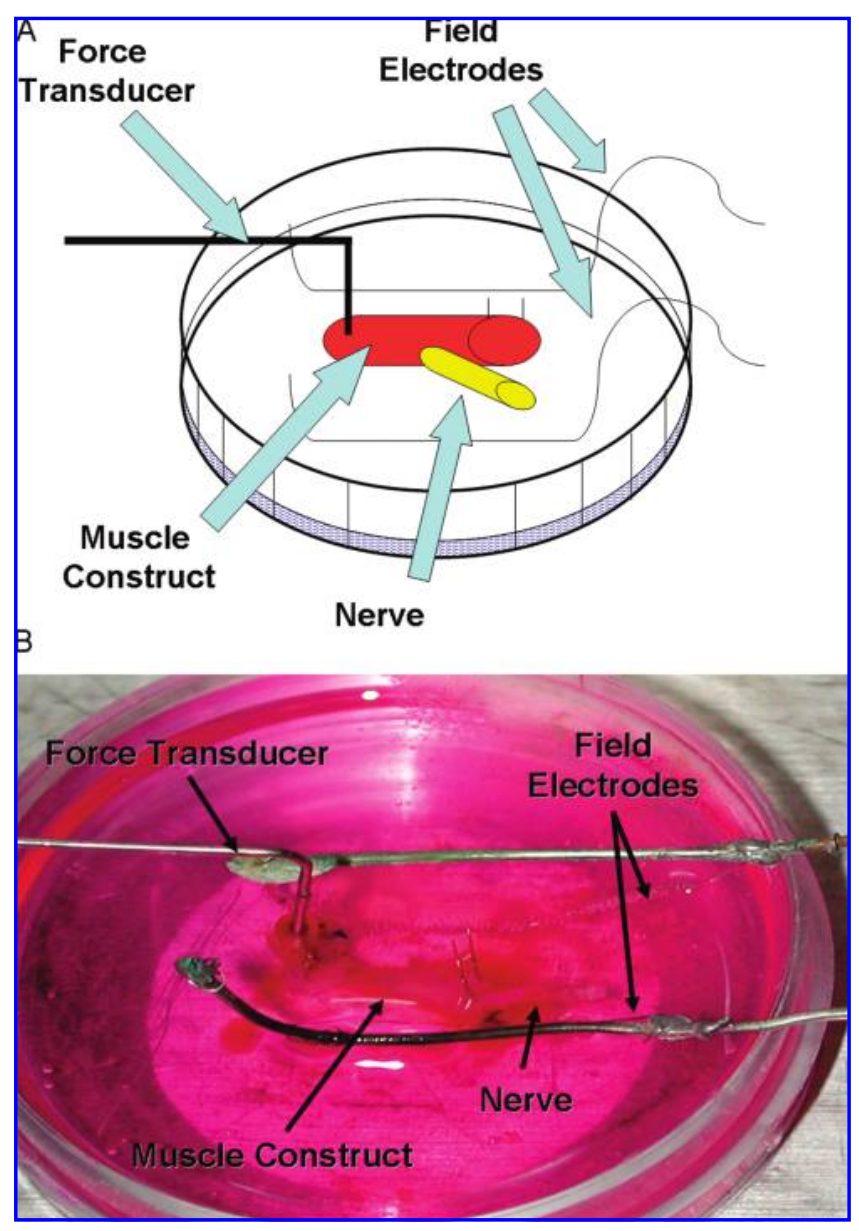

FIG. 2. (A). Drawing of a skeletal muscle construct after harvest from the rat in preparation for direct (field) stimulation. The tissueengineered construct is placed in a tissue culture dish with culture medium. The muscle construct is pinned to the dish on one end (right side), and the other end of the muscle is attached to a force transducer arm. Field stimulation electrodes are placed along both sides, parallel to the muscle. Upon electrical stimulation, the muscle contracts in a linear fashion, and the force transducer measures the force produced. (B) Direct (field) stimulation of the skeletal muscle construct. The muscle is pinned to the dish at the right. Note the force transducer, field electrodes, and nerve. Color images available online at www.liebertpub.com/ten. 


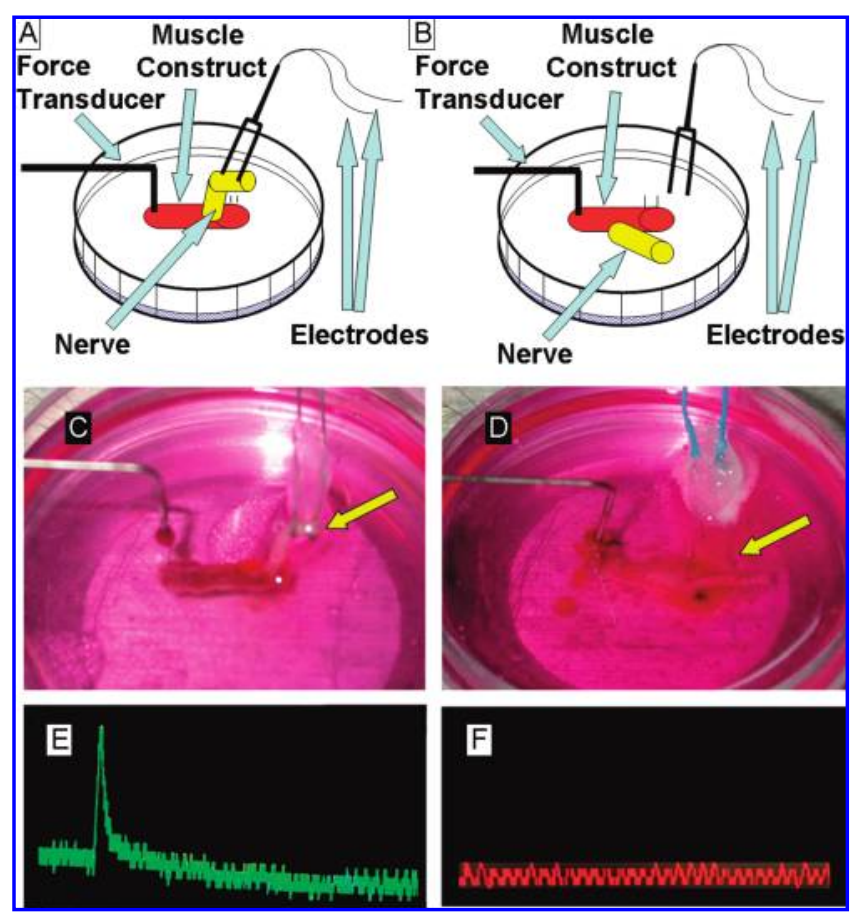

FIG. 3. Indirect (via the nerve) skeletal muscle construct stimulation. (A) Drawing of a skeletal muscle construct in growth medium with electrodes placed directly into the nerve. The muscle construct is still pinned to the dish at the right and connected to the force transducer at the left. (B) Drawing of the skeletal muscle construct with the electrodes removed from the nerve and placed at an equidistant point from the muscle construct as in the previous indirect stimulation. (C) Neurotized skeletal muscle construct pinned to a culture dish and attached to the force transducer. Field stimulation wires are removed and replaced with electrodes that are inserted directly into the nerve (arrow). (D) Electrodes placed at an equidistant location from the muscle construct but not touching the nerve to exclude the possibility that direct field stimulation, rather than indirect stimulation via the nerve, resulted in muscle contraction. (E) Force tracing after indirect stimulation with electrode touching nerve. (F) Force tracing after electrical stimulation with electrode not touching nerve but at equidistant point from muscle construct. Color images available online at www.liebertpub.com/ten.

the muscle via NMJs and not solely due to the nerve acting as physical electrical conduit, the electrodes were again placed touching the nerve. Before repeat indirect stimulation, curare (tubocurarine chloride hydrate, Sigma), a potent NMJ inhibitor, was dissolved in a buffered physiological salt solution (137 mM sodium chloride, $24 \mathrm{mM}$ sodium bicarbonate, $11 \mathrm{mM}$ glucose, $5 \mathrm{mM}$ potassium chloride, $2 \mathrm{mM}$ calcium chloride, $1 \mathrm{mM}$ magnesium sulfate, $1 \mathrm{mM}$ sodium dihydrogen phosphate, and $0.024 \mathrm{mM}$ tubocurarine chloride). ${ }^{15}$ The curare solution was added to the muscle bath solution, and the electrical stimulation and contraction recording were then repeated.

\section{Histological assessment}

After functional assessment, the skeletal muscle constructs were embedded in tissue freezing medium (Triangle Biomedical Sciences, Durham, NC), frozen in liquid nitrogen, and stored at $-80^{\circ} \mathrm{C}$. The frozen constructs were sectioned in a $-30^{\circ} \mathrm{C}$ cryotome and placed on glass slides. The sections were then fixed in cold acetone. Sections of neurotized and non-neurotized muscle constructs were routinely stained with hematoxylin and eosin and evaluated for the presence of NMJs using an immunostaining technique using alpha-bungarotoxin, a substance known to bind to the neuromuscular nicotinic acetylcholine receptors of NMJs. For alpha-bungarotoxin staining, the previously sectioned, frozen slides were allowed to thaw for $30 \mathrm{~min}$ at room temperature then fixed in $100 \%$ methanol at $-20^{\circ} \mathrm{C}$ for $10 \mathrm{~min}$. PBS was pipetted onto the slide for $3 \mathrm{~min}$ and removed. The fluorescein isothiocyanate-labeled alphabungarotoxin (Sigma-Aldrich, St. Louis, MO) was diluted, $15 \mu \mathrm{L}$ in $1.5 \mathrm{~mL}$ of PBS, and added to the slides. After $5 \mathrm{~min}$, the alpha-bungarotoxin-PBS was removed, and the slides were rinsed with PBS 5 times. Then 4',6-diamidino2-phenylindole (Sigma-Aldrich) counterstain was added to each slide, a cover slip was placed over the sectioned tissue, and the tissues were visualized using an immunofluorescent microscope. Digital images were taken with an Olympus DP70 digital camera and associated software through an Olympus BX 51 microscope (Hamburg, Germany).

\section{Statistical analysis}

The mean stimulation contraction force value, standard errors, and one-way analysis of variance were calculated for each implantation site. A $p<0.05$ was considered significant.

\section{RESULTS}

After 4 weeks of in vivo implantation, all 8 chambers were explanted (Fig. 1E, F). It was found that the chambers were filled with viable tissue occupying their entire volume. The femoral vessels had incorporated into the newly formed tissue and remained intact and patent. At the neurotized construct sites, the proximal stumps of the femoral nerves were incorporated into the constructs.

\section{Functional results}

Direct muscle field stimulation generated visible contractions in the construct and measurable force in all 8 constructs tested, including those with or without nerve stump implants. Force values for the control skeletal muscle constructs demonstrated an average peak tetanic force of $124 \pm 31 \mu \mathrm{N}$, which is consistent with our earlier published data on non-neurotized, vascularized skeletal muscle constructs. ${ }^{3}$ The average tetanic forces generated by the 


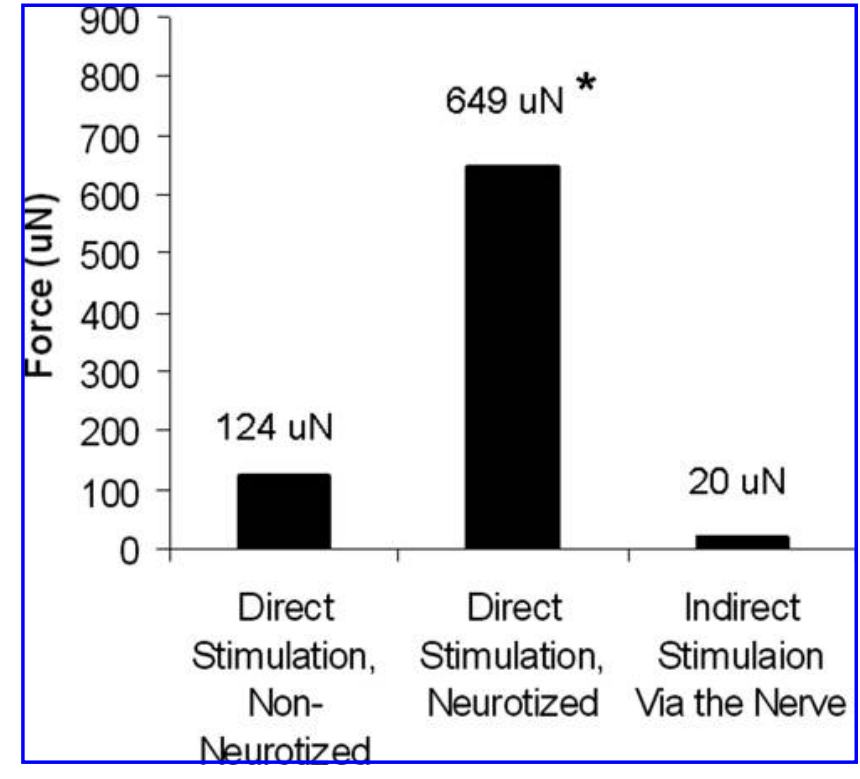

FIG. 4. Average peak force. There was a statistical difference between the average tetanic force of non-neurotized skeletal muscle controls $(124 \pm 31 \mu \mathrm{N})$ and the average tetanic force of neurotized skeletal muscle constructs $(649 \pm 228 \mu \mathrm{N})$ via direct (field) stimulation $(p<0.05)$. Average twitch force of neurotized skeletal muscle constructs via indirect stimulation by the nerve was $20.4 \mu \mathrm{N}$. The direct forces were obtained during tetany, whereas the indirect force via the nerve was obtained during twitch stimulation.

neurotized skeletal muscle constructs in response to direct field stimulation averaged $649 \pm 228 \mu \mathrm{N}(p<0.05$, Fig. 4), more than 5 times as great as at the non-neurotized control sites.

After the direct field stimulation tests, indirect stimulation of a neurotized construct was performed by placing needle electrodes in contact with the nerve (Fig. 3C). With indirect electrical stimulation, the experimental, neurotized skeletal muscle construct generated measurable linear force contractions (Fig. 3E), producing an average of $20.4 \mu \mathrm{N}$ of twitch force (Fig. 4). Immediately after indirect stimulation, the electrodes were removed from the nerve and placed in the bath at a point equidistant from the muscle construct to determine whether the force produced was due to field stimulation rather than indirect stimulation down the nerve. With electrical stimulation in this configuration, no force was elicited (Fig. 3F). Next, the electrodes were replaced into the nerve, and stimulated contraction was again confirmed. Lastly, without disturbing the construct, nerve, or electrodes, curare, a potent neuromuscular junction inhibitor was added to the bath. Repeat indirect stimulation elicited no force from any of the neurotized constructs.

\section{Histological results}

Hematoxylin and eosin staining demonstrated results similar to those previously reported, including the presence

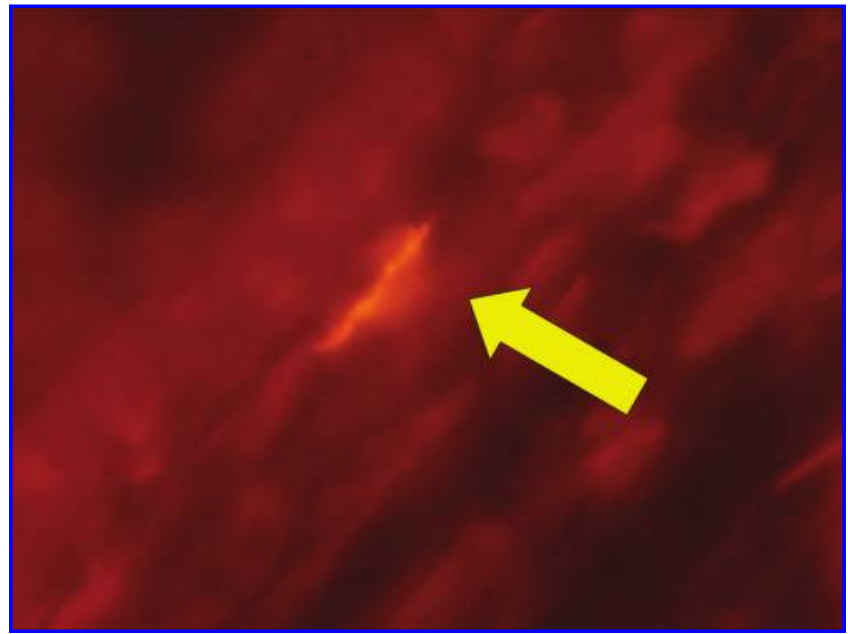

FIG. 5. Example of neuromuscular junction formation (arrow) in a neurotized skeletal muscle construct stained using alphabungarotoxin $(100 \times)$. Color images available online at www liebertpub.com/ten.

of surviving skeletal muscle cells interspersed with collagen deposition and robust angiogenesis sprouting from the femoral vessels. Examination of sections from neurotized constructs immunostained with the alpha-bungarotoxin technique identified rare NMJ presence (Fig. 5). A comprehensive analysis of the tissues in an attempt to quantify NMJ's was not performed. This study surveyed random sections to identify the presence of NMJ's. No NMJs were identified in the non-neurotized controls.

\section{DISCUSSION}

Several tissue-engineering techniques have been used in the effort to produce skeletal muscle. Researchers have created muscle tissue using in vitro "self-assembly" techniques $^{5,13,16-18}$ and in vivo implantation techniques. $3,4,19$ Additionally, skeletal muscle constructs have been produced by seeding myoblasts onto scaffolds to achieve 3-D tissues in vitro ${ }^{20-24}$ and in vivo. ${ }^{25-28}$ These scaffolds have been composed of a variety of materials, including poly-Llactic acid/polylactic-glycolic acid sponges, ${ }^{26}$ acellularized skeletal muscle, ${ }^{20,27}$ polyglycolic acid meshes, ${ }^{25,28}$ and suspensions of alginate or hyaluronic acid hydrogels. ${ }^{25}$ Significant research has also been undertaken to understand the properties required to optimize the scaffolds, such as material chemistry and roughness, porosity, wettability, and architecture and how these elements best interact with tissue properties such as cell-adhesion ability. ${ }^{29-31}$

Diffusion characteristics of oxygen, nutrients, and waste metabolites of tissues are ultimately what determine the size of a construct that can be viably sustained. In the attempts to generate engineered tissue of any size much greater than 
$200 \mu \mathrm{m}$ in thickness, ${ }^{32}$ substantial efforts have been devoted in the field of tissue engineering to the vascularization of these tissue constructs in vitro ${ }^{26}$ and in vivo. ${ }^{4,33,34}$ In vivo vascularization can be augmented by creating tissue that generate angiogenic factors such as vascular endothelial growth factor, platelet-derived growth factor, endothelium-derived growth factor, recombinant human growth hormone, and transforming growth factor-beta using genetically engineered cells that produce the protein of interest. ${ }^{35-38}$ Conversely, vascularization can be induced via a spontaneous model of angiogenesis. ${ }^{3,4,19,33,34}$

Our initial attempts at tissue engineering a vascularized skeletal muscle construct in vivo stemmed from an adaptation of a method of inducing spontaneous angiogenesis from an arteriovenous vascular pedicle, which, when contained within a "chamber," could provide a well-vascularized platform to support the survival and growth of 3-D tissues. ${ }^{4}$ We demonstrated the development of axially vascularized skeletal muscle constructs in vivo in the rat, which exhibited histological and contractile properties similar to those of native muscle. The observation of numerous myotubes present within the constructs that stained positively for the muscle-specific intermediate-filament protein desmin provided evidence of differentiation of implanted myocytes. Also, a striated banding pattern similar to that in skeletal muscle was identified. Physiological properties, namely force measurements, of these constructs also resembled those of developing muscles. ${ }^{3}$ However, the ultimate force produced was much lower than that of native skeletal muscle (35.4 \pm $62.2 \mathrm{~N} / \mathrm{m}^{2}$ vs $239.7 \pm 19.1 \mathrm{kN} / \mathrm{m}^{2}$ ) for mouse extensor digitorum longus muscle. ${ }^{20}$

We hypothesized that providing neural input to developing tissue-engineered skeletal muscle constructs would improve their contractile forces and allow for their indirect stimulation. The neural influence on the development of skeletal muscle fibers, on which the hypothesis of this article is based, is well documented. The relationship between muscle types and motor nerves was established in early studies. ${ }^{39}$ Skeletal muscle properties and the effects of innervation have been studied using many methods, including denervation, ${ }^{40,41}$ and it has been shown that, with denervation, fast and slow muscle will start to transform into a phenotypically slow muscle. Classic cross-reinnervation studies have shown that, when the fast flexor digitorum longus muscle was cross-reinnervated with a slow tibial nerve, twitch contraction times slowed 50\%, had low adenosine triphosphatase activity, and reacted with slow myosin antibodies only. In contrast, when the soleus muscle was cross-reinnervated with a fast flexor digitorum longus nerve, the contraction time almost doubled, but there were no changes in adenosine triphosphatase activity, and there was mixed reactivity to fast and slow antibodies. ${ }^{42}$ In similar studies, when the slow-twitch semimembranosus proprius muscle of the rabbit was crossreinnervated with a fast nerve, almost all fibers switched to a fast phenotype. ${ }^{43}$
Our findings support our hypothesis that neurotization of skeletal muscle would increase force-generating capabilities. Specifically, we found that neurotization of developing skeletal muscle constructs in vivo resulted in a 5-fold increase in average peak tetanic force over that of nonneurotized constructs ( 649 vs $124 \mathrm{mN}, p<0.05$ ). In addition, stimulation of the muscle construct indirectly via the nerve was successful, eliciting characteristic force tracing, albeit at a lower twitch force. Upon the addition of curare, indirect stimulation of the muscle construct ceased producing contractions. Taken together, these observations provide physiologic evidence that NMJs developed within the neurotized constructs. The histologic demonstration of NMJs on immunostaining further bolster this conclusion.

There are several possible explanations for the low forcegenerating capability of the construct via indirect versus direct stimulation. One possibility is that only a few axons in the short experimental time of 4 weeks physically make contact with the muscle. Another possibility is that the muscle cells that have NMJs do not have well-established intracellular junctions with other myocytes and therefore that few myocytes receive excitation via indirect stimulation.

A limitation of this study was the ex vivo functional assessment of the skeletal muscle constructs. It would be possible to stimulate the nerve in vivo, and typically this is the preferred method to stimulate muscle. However, our constructs do not have an origin or insertion point and therefore are not attached to any fixed structure. By explanting the construct, we were able to fix one end to a stationary surface and the other end to the force transducer and obtain reliable force measurements. By explanting the constructs and performing the force testing ex vivo, we may obtain suboptimal performance secondary to adenosine triphosphate depletion, electrolyte imbalances, and other things, because of detachment from their intrinsic blood supply. However, the muscle tissue in the neurotized constructs and the non-neurotized controls should have been affected similarly. Because the experiment was designed only to test the difference between the neurotized and non-neurotized constructs, we felt that the ex vivo testing was an acceptable method for this initial study.

In this work, we have succeeded in generating in vivo neurotized, vascularized skeletal muscle constructs. Through vascularization, we have been able to create larger skeletal muscle constructs, and now, with neurotization, we have been able to increase their contractile forces substantially. Additionally, with neurotization, we were able to increase the muscle constructs' function by being able to direct stimulation and contraction. With the generation of this vascularized, neurotized muscle construct, we are one step closer to engineering a transplantable tissue flap that can be transferred microvascularly via an axial blood supply and have the ability to achieve directed muscular contractions via nerve connections.

Future studies will focus on differential neurotization of tissue-engineered skeletal muscle and how this may affect 
phenotypic maturation and differentiation. Molecular analysis of the skeletal muscle after maturation will be performed to evaluate myosin heavy chain isoform differences between these groups. Future experiments, using immunofluorescentlabeled cultured skeletal myocytes, will also assist in the analysis of the distribution and quantification of the engineered skeletal muscle tissue and its relationship to NMJs.

\section{ACKNOWLEDGMENTS}

The authors thank Dr. Tatiana Kostrominova, Ph.D., and Dr. Jacob Vandermeulen, Ph.D., for their expert advice on the histological evaluation of samples presented in this study. The study was supported in part by a Plastic Surgery Educational Foundation grant.

\section{REFERENCES}

1. Langer R, Vacanti JP. Tissue engineering. Science 260, 920, 1993.

2. Birla RK, Borschel GH, Dennis RG, Brown DL. Myocardial engineering in vivo: formation and characterization of contractile, vascularized three-dimensional cardiac tissue. Tissue Eng 11, 803, 2005.

3. Borschel, GH, Dow DE, Dennis RG, Brown DL. Tissueengineered axially vascularized contractile skeletal muscle. Plast Reconstr Surg 117, 2235, 2006.

4. Cronin KJ, Messina A, Knight KR, Cooper-White JJ, Stevens GW, Penington AJ, et al. New murine model of spontaneous autologous tissue engineering, combining an arteriovenous pedicle with matrix materials. Plast Reconstr Surg 113, 260 , 2004.

5. Dennis RG, Kosnik PE. Excitability and isometric contractile properties of mammalian skeletal muscle constructs engineered in vitro. In Vitro Cell Dev Biol Anim 36, 327, 2000.

6. Hattori Y, Doi K, Fuchigami Y, Abe Y, Kawai S. Experimental study on donor nerves for brachial plexus injury: comparison between the spinal accessory nerve and the intercostal nerve Plast Reconstr Surg 100, 900, 1997.

7. Nehrer-Tairych GV, Rab M, Kamolz L, Deutinger M, Stohr HG, Frey M. The influence of the donor nerve on the function and morphology of a mimic muscle after cross innervation: an experimental study in rabbits. Br J Plast Surg 53, 669, 2000.

8. Jevsek M, Mars T, Mis K, Grubic Z. Origin of acetylcholinesterase in the neuromuscular junction formed in the in vitro innervated human muscle. Eur J Neurosci 20, 2865, 2004.

9. Kobayashi T, Askanas V. Acetylcholine receptors and acetylcholinesterase accumulate at the nerve-muscle contacts of de novo grown human monolayer muscle cocultured with fetal rat spinal cord. Exp Neurol 88, 327, 1985.

10. Mis K, Mars T, Jevsek M, Strasek H, Golicnik M, Brecelj J, et al. Expression and distribution of acetylcholinesterase among the cellular components of the neuromuscular junction formed in human myotube in vitro. Chem Bio Interact 29, 157, 2005.
11. Mars T, King MP, Miranda AF, Walker WF, Mis K, Grubic Z. Functional innervation of cultured human skeletal muscle proceeds by two modes with regard to agrin effects. Neuroscience 118, 87, 2003.

12. Institute of Laboratory Animal Research, Commission on Life Sciences, National Research Council. Guide for the Care and Use of Laboratory Animals. Washington, D.C.: National Academy Press, 1996.

13. Huang YC, Dennis RG, Larkin L, Baar, K. Rapid formation of functional muscle in vitro using fibrin gels. J Appl Physiol 98, 706, 2005.

14. Borgens RB, Shi R. Immediate recovery from spinal cord injury through molecular repair of nerve membranes with polyethylene glycol. FASEB J 14, 27, 2000.

15. Brooks SV, Opiteck JA, Faulkner JA. Conditioning of skeletal muscles in adult and old mice for protection from contractioninduced injury. J Gerontol A Biol Sci Med Sci 56A, B163, 2001.

16. Dennis RG, Kosnik PE, Gilbert ME, Faulkner JA. Excitability and contractility of skeletal muscle engineered from primary cultures and cell lines. Am J Physiol Cell Physiol 280, C288, 2001.

17. Kosnik PE, Faulkner JA, Dennis RG. Functional development of engineered skeletal muscle from adult and neonatal rats. Tissue Eng 7, 573, 2001.

18. Strohman RC, Bayne E, Spector D, Obinata T, MicouEastwood J, Maniotis A. Myogenesis and histogenesis of skeletal muscle on flexible membranes in vitro. In Vitro Cell Dev Biol 26, 201, 1990.

19. Messina A, Bortolotto SK, Cassell OC, Kelly J, Abberton KM, Morrison, WA. Generation of a vascularized organoid using skeletal muscle as the inductive source. FASEB J 19, 1570, 2005.

20. Borschel GH, Dennis RG, Kuzon, WM JR. Contractile skeletal muscle tissue-engineered on an acellular scaffold. Plast Reconstr Surg 113, 595, 2004.

21. Fuchs JR, Pomerantseva I, Ochoa ER, Vacanti JP, Pauza DO Fetal tissue engineering: in vitro analysis of muscle constructs. J Pediatr Surg 38, 1348, 2003.

22. Neumann T, Hauschka SD, Sanders JE. Tissue engineering of skeletal muscle using polymer fiber arrays. Tissue Eng $\mathbf{9}$, 1003, 2003.

23. Shen JY, Chan-Park MB, Feng ZQ, Chan V, Feng ZW. UVembossed microchannel in biocompatible polymeric film: application to control of cell shape and orientation of muscle cells. J Biomed Mater Res B Appl Biomater 77, 423, 2006.

24. Vandenburgh HH, Karlisch P, Farr L. Maintenance of highly contractile tissue-cultured avian skeletal myotubes in collagen gel. In Vitro Cell Dev Biol 24, 166, 1993.

25. Kamelger FS, Marksteiner R, Margreiter E, Klima G, Wechselberger G, Hering $\mathrm{S}$, et al. A comparative study of three different biomaterials in the engineering of skeletal muscle using a rat animal model. Biomaterials 25, 1649, 2004.

26. Levenberg S, Rouwkema J, Macdonald M, Garfein ES, Kohane DS, Darland, et al. Engineering vascularized skeletal muscle tissue. Nat Biotechnol 23, 879, 2005.

27. Marzaro M, Conconi MT, Perin L, Giuliani S, Gamba P, De Coppi, et al. Autologous satellite cell seeding improves in vivo 
biocompatibility of homologous muscle acellular matrix implants. Int J Mol Med 10, 177, 2002.

28. Saxena AK, Willital GH, Vacanti JP. Vascularized threedimensional skeletal muscle tissue-engineering. Biomed Mater Eng 11, 275, 2001.

29. Karande TS, Ong JL, Agrawal CM. Diffusion in musculoskeletal tissue engineering scaffolds: design issues related to porosity, permeability, architecture, and nutrient mixing. Ann Biomed Eng 32, 1728, 2004.

30. Lim JY, Donahue HJ. Biomaterial characteristics important to skeletal tissue engineering. J Musculoskelet Neuronal Interact 4, 396, 2004.

31. Musuko T, Iwasaki N, Yamane S, Funakoshi T, Majima T, Minami A, et al. Chitosan-RGDSGGC conjugate as a scaffold material for musculoskeletal tissue engineering. Biomaterials 26, 5339, 2005.

32. Colton CK. Implantable biohybrid artificial organs. Cell Transplant 4, 415, 1995.

33. Mian R, Morrison WA, Hurley JV, Penington AJ, Romeo R, Tanaka $\mathrm{Y}$, et al. Formation of new tissue from an arteriovenous loop in the absence of added extracellular matrix. Tissue Eng 6, 595, 2000.

34. Tanaka Y, Tsutsumi A, Crowe DM, Tajima S, Morrison WA. Generation of an autologous tissue (matrix) flap by combining an arteriovenous shunt loop with artificial skin in rats: preliminary report. Br J Plast Surg 53, $51,2000$.

35. Lu Y, Shansky J, Del Tatto M, Ferland P, Wang X, Vandenburgh H. Recombinant vascular endothelial growth factor secreted from tissue-engineered bioartificial muscles promotes localized angiogenesis. Circulation 104, 594, 2001.

36. Lu Y, Shansky J, Del Tatto M, Ferland P, McGuire S, Marszalkowski J, et al. Therapeutic potential of implanted tissue-engineered bioartificial muscles delivering recombinant proteins to the sheep heart. Ann N Y Acad Sci 961, 78, 2002.
37. Powell C, Shansky J, Del Tatto M, Forman DE, Hennessey J, Sullivan $\mathrm{K}$, et al. Tissue-engineered human bioartificial muscles expressing a foreign recombinant protein for gene therapy. Hum Gene Ther 10, 565, 1999.

38. Springer ML, Ozawa CR, Banfi A, Kraft PE, Ip TK, Brazelton, et al. Localized arteriole formation directly adjacent to the site of VEGF-induced angiogenesis in muscle. Mol Ther 7, 441, 2003.

39. Buller AJ, Eccles JC, Eccles RM. Differentiation of fast and slow muscles in the cat hind limb. J Psychol 150, 399, 1960.

40. d'Albis A, Goubel F, Couteaux R, Janmot C, Mira JC. The effect of denervation on myosin isoform synthesis in rabbit slow-type and fast-type muscles during terminal differentiation. Denervation induces differentiation into slow-type muscles. Eur J Biochem 223, 249, 1994.

41. Leeuw T, Kapp M, Pett D. Role of innervation for development and maintenance of troponin subunit isoform patterns in fast and slow-twitch muscles of the rabbit. Differentiation $\mathbf{5 5}$, 193, 1994.

42. Gauthier GF, Burke RE, Lowey S, Hobbs AW. Myosin isozymes in normal and cross-reinnervated cat skeletal muscle fibers 97, 756, 1983.

43. Bacou F, Rouanet P, Barjot C, Janmot C, Vigneron P, et al. Expression of myosin isoforms in dennervated, crossreinnervated, and electrically stimulated rabbit muscles. Eur J Biochem 236, 539, 1996.

Address reprint requests to: David L. Brown, M.D. 2130 Taubman Center 1500 E Medical Center Drive Ann Arbor, MI 48109

E-mail: davbrown@umich.edu 

This article has been cited by:

1. D. E. Discher, D. J. Mooney, P. W. Zandstra. 2009. Growth Factors, Matrices, and Forces Combine and Control Stem Cells. Science 324:5935, 1673-1677. [CrossRef]

2. Merel Koning, Martin C. Harmsen, Marja J. A. van Luyn, Paul M. N. Werker. 2009. Current opportunities and challenges in skeletal muscle tissue engineering. Journal of Tissue Engineering and Regenerative Medicine n/a-n/a. [CrossRef] 